\title{
Optimal design of active EMC filters
}

\author{
B. Chand, T. Kut, and S. Dickmann \\ Fundamentals of Electrical Engineering, Helmut Schmidt University / University of the Federal Armed Forces Hamburg, \\ Germany \\ Correspondence to: B. Chand (benian.chand@hsu-hh.de)
}

\begin{abstract}
A recent trend in automotive industry is adding electrical drive systems to conventional drives. The electrification allows an expansion of energy sources and provides great opportunities for environmental friendly mobility. The electrical powertrain and its components can also cause disturbances which couple into nearby electronic control units and communication cables. Therefore the communication can be degraded or even permanently disrupted. To minimize these interferences, different approaches are possible. One possibility is to use EMC filters. However, the diversity of filters is very large and the determination of an appropriate filter for each application is time-consuming. Therefore, the filter design is determined by using a simulation tool including an effective optimization algorithm. This method leads to improvements in terms of weight, volume and cost.
\end{abstract}

\section{Introduction}

The EMC of electronic systems is often achieved by filter networks. The used components take an increasing part of the total system weight and volume. Therefore, the optimal filter for different requirements is determined with previous simulation estimations. The filters are characterized in terms of the number and type of filter stages, component values or attenuation. Therefore passive, active and hybrid filter structures are used. The active filters are investigated by using a feedforward structure (Fig. 1). Furthermore, the frequency characteristic of the filter is described by the use of A-matrices. The existing common- and differential-mode interferences are attenuated by an interconnection of common- and differential-mode filters. The input and output impedances are important design criteria. Taking into account these criteria, the manual design is very timeconsuming due to the large number of possible filter structures. Therefore, a algorithm was implemented which determines the optimal filter network depending on the required parameters. To determine the optimal filter for given boundary conditions the Particle Swarm Optimization (PSO) process is used. The output of the software includes not only the filter network topology and the component values, but also a comparison of the cost, weight and the simulation time. These results are verified by measurements. Finally, the determined filter is studied in a real, practical construction of an electrical powertrain.

\section{Concepts of reducing the interferences by using EMC filters}

We consider filter circuits which attenuate conducted interference. Three groups of filter circuits are explained in this chapter: Passive, active and hybrid filters for common-mode and differential-mode. Parasitic effects are not taken into account. Another topic of this section is the description of a two-port network by an A-matrix. With these methods it is possible to determine the frequency characteristics of an electric filter circuit in a short time.

\section{Passive filters}

are the simplest form of electric filters (Hagmann, 2008). Since the components require no power supply, the combination of these components are called passive filters. In this case, the filter should attenuate harmonics. For this reason, a low-pass topology will be used.

\section{Active filters}

reduce the emitted interference from the source by means of controllable active components (Schinkel, 2009). The conducted interference is minimized by means of a positive feedback structure, see Fig. 1. In that case, the signal including 


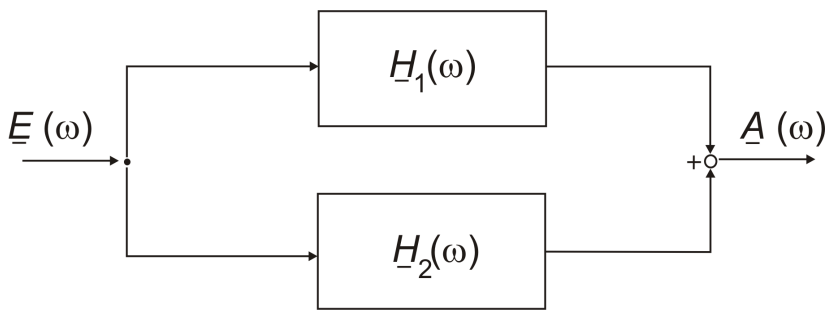

Fig. 1. Positive feedback structure for active filtering.

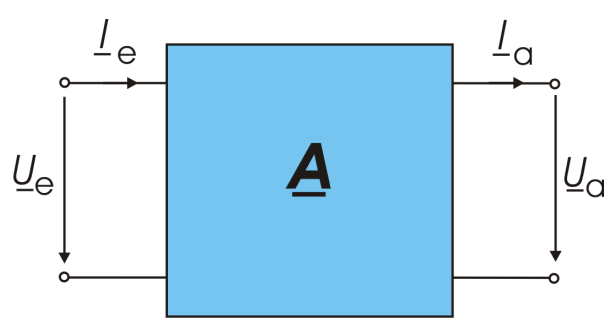

Fig. 2. Schematic description of a four-terminal network.

the interference $E(\omega)$ is conducted in parallel over the functions $\underline{H}_{1}(\omega)$ and $\underline{H}_{2}(\omega)$, so the unwanted interference can be canceled and the required output signal $\underline{A}(\omega)$ is produced.

\section{Hybrid filters}

circuits consist of active and passive filter circuits. The advantage with such a configuration is the attenuation of the interference to a large amount by means of an active filter. Therefore, the passive components can be designed smaller, which can add to weight and cost savings.

\section{Description of the filter using A-Matrices}

To determine the frequency characteristic of a filter circuit, A-matrices can be used. In Fig. 2 a four-terminal network with its connections, the output and input current and the output and input voltage is shown.

The system can be represented using a matrix notation:

$$
\left(\begin{array}{l}
\underline{U}_{\mathrm{e}} \\
\underline{I}_{\mathrm{e}}
\end{array}\right)=\underline{\mathbf{A}} \cdot\left(\begin{array}{l}
\underline{U}_{\mathrm{a}} \\
\underline{I}_{\mathrm{a}}
\end{array}\right) \text { with } \underline{\mathbf{A}}=\left(\begin{array}{ll}
\underline{A}_{11} & \underline{A}_{12} \\
\underline{A}_{21} & \underline{A}_{22}
\end{array}\right)
$$

The individual elements of the matrix $\underline{\mathbf{A}}$ are:

$$
\begin{aligned}
& \underline{A}_{11}=\left.\frac{\partial \underline{U}_{\mathrm{e}}}{\partial \underline{U}_{\mathrm{a}}}\right|_{\underline{I}_{\mathrm{a}}=0} \underline{A}_{12}=\left.\frac{\partial \underline{U}_{\mathrm{e}}}{\partial \underline{I}_{\mathrm{a}}}\right|_{\underline{U}_{\mathrm{a}}=0} \\
& \underline{A}_{21}=\left.\frac{\partial \underline{I}_{\mathrm{e}}}{\partial \underline{U}_{\mathrm{a}}}\right|_{\underline{I}_{\mathrm{a}}=0} \underline{A}_{22}=\left.\frac{\partial \underline{I}_{\mathrm{e}}}{\partial \underline{I}_{\mathrm{a}}}\right|_{\underline{\mathrm{a}}_{\mathrm{a}}=0}
\end{aligned}
$$

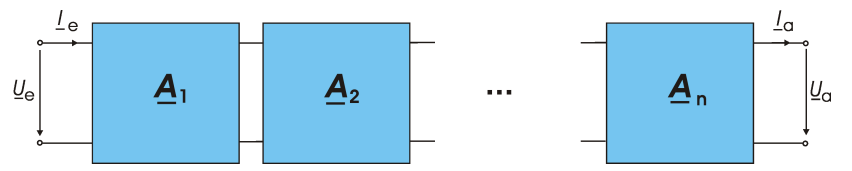

Fig. 3. Series connection of $\underline{\mathbf{A}}$-matrices.

The advantage for a series connection of A-matrices, as described in Fig. 3, is the possibility to multiply the individual matrices easily. The product results in an overall matrix. For the given overall matrix $\mathrm{A}_{\text {total }}$, it is now possible to determine the ratio of the input to output voltage. It follows from the inverse of the matrix element $\underline{A}_{11}$ (Marti, 2007).

$\left(\begin{array}{l}\underline{U}_{\mathrm{e}} \\ \underline{I}_{\mathrm{e}}\end{array}\right)=\underline{\mathrm{A}}_{1} \cdot \underline{\mathrm{A}}_{2} \cdot \underline{\mathrm{A}}_{3} \cdot \ldots \cdot \underline{\mathrm{A}}_{\mathrm{n}-1} \cdot \underline{\mathrm{A}}_{\mathrm{n}} \cdot\left(\begin{array}{l}\underline{U}_{\mathrm{a}} \\ \underline{I}_{\mathrm{a}}\end{array}\right)$

$\left(\begin{array}{l}\underline{U}_{\mathrm{e}} \\ \underline{I}_{\mathrm{e}}\end{array}\right)=\underline{\mathrm{A}}_{\mathrm{tot}} \cdot\left(\begin{array}{l}\underline{U}_{\mathrm{a}} \\ \underline{I}_{\mathrm{a}}\end{array}\right)$

For later considerations of active and hybrid filters the $\underline{\mathbf{A}}$ matrix of the active filter results from Fig. 5 (Rehfeld, 2012):

$\underline{\mathbf{A}}=\left(\begin{array}{cc}\frac{s^{2} \cdot k_{1} \cdot L_{1} \cdot C_{1}+s \cdot C_{1} \cdot R_{\mathrm{C}}+1}{s^{2} \underline{k}_{2} \cdot L_{1} \cdot C_{1}+s \cdot C_{1} \cdot R_{\mathrm{C}}+1} & \frac{s \cdot L_{1} \cdot\left(s \cdot C_{1} \cdot R_{\mathrm{C}} 1\right)}{\underline{s}^{2} \cdot L_{1} \cdot C_{1}\left(\underline{\kappa} \cdot(1+v)+\frac{v}{u}\right)+s \cdot C_{1} \cdot R_{\mathrm{C}}+1} \\ \frac{s \cdot C_{1}(1-\underline{\kappa}(1+v))}{s^{2} \cdot \underline{k}_{2} \cdot L_{1} \cdot C_{1}+s \cdot C_{1} \cdot R_{\mathrm{C}}+1} & 1\end{array}\right)$

with $k_{1}=1+\frac{v}{\ddot{u}}, \underline{k}_{2}=\underline{\kappa} \cdot(v+1)+\frac{v}{\ddot{u}}, \underline{\kappa}=\frac{\underline{U}_{\mathrm{OP}}}{\underline{U}_{\mathrm{e}}}, v=\frac{R_{2}}{R_{1}}$, $\ddot{u}=\sqrt{\frac{L_{1}}{L_{2}}}, s=j \cdot 2 \cdot \pi \cdot f$

\section{Common-mode and differential-mode filters}

Since interferences are often composed of common-mode and differential-mode interferences, a seperate consideration of common-mode and differential-mode filters is recommended. This allows a more effective attenuation of the interferences. X-capacitors or differential-mode chokes are used as classical elements for reduction of differential-mode interferences. In DC and single phase systems, X-capacitors are located between the supply and the return conductor. Common-mode filters attenuate common-mode interferences and represent at the same time short circuit for differentialmode interferences. The main components of this filter group are common-mode inductors and Y-capacitors. The leakage currents through the Y-capacitor should be kept small, because otherwise the capacitor bypasses the insulation. According to (DIN-EN, 50178), depending on the model, leakage currents of $0.75 \mathrm{~mA}$ to $3.5 \mathrm{~mA}$ are allowed. Therefore for the filter design, two different simulations are studied. One for the differential-mode filter and one for the commonmode filter. Thereby the common-mode filter is simulated for 


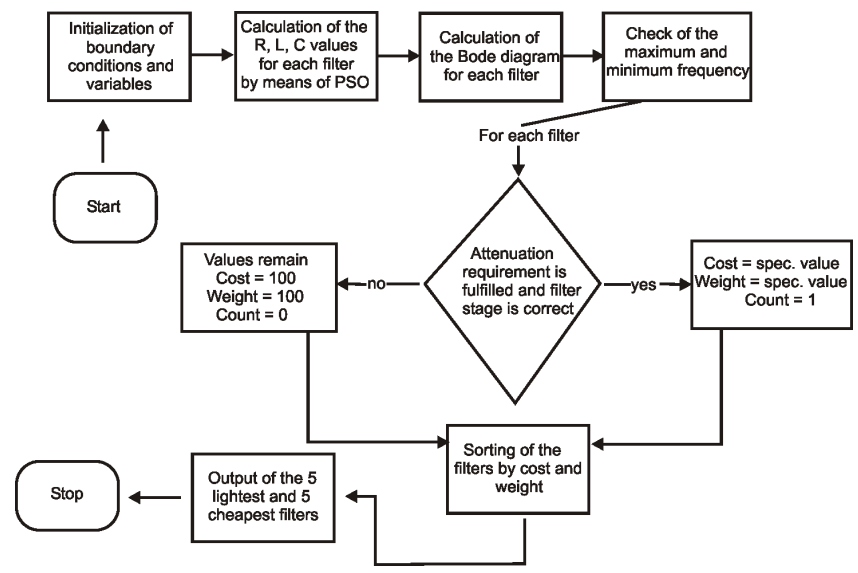

Fig. 4. Program Flowchart.

one wire and the ground and the same common-mode filter is used for the second wire as well. So the approach of $2 \times 2$ A-matrices can be used here.

\section{Design algorithm for EMC filters}

\subsection{Program flow of the simulation script}

A program for designing electrical filters has been created. After entering the required attenuation in a certain frequency band, the script displays the cheapest and lightest filter topology for given boundary conditions. In this section, the algorithm of the script will be introduced and some important details are discussed.

With the help of a graphical user interface (GUI) the boundary conditions for the configuration of the filter circuit are set. These are the minimum and maximum values of the used inductors, capacitors and resistors $\left(L_{\min }, L_{\max }, C_{\min }\right.$, $\left.C_{\max }, R_{\min }, R_{\max }\right)$. Another input parameter is the attenuation within a given frequency band. Furthermore, it should be determined, whether there is a high ohmic or a low ohmic input and output impedance, because they influence the behavior of the filter circuit. Moreover, it is necessary to determine the number of the filter stages. For the evaluation, the program needs an input signal described in a text file. As output parameter of the script the required computing time or the calculated attenuation can be selected. In Fig. 4, the program flowchart of the main program is shown.

First of all, the entered boundary conditions are stored and initialized. In the next step, the various components of the different filter circuits are calculated. This is done using an optimization algorithm which is called Particle Swarm Optimization (PSO) (Brodersen, 2006). This process will be discussed later in this section. After calculating the components of each filter, the frequency characteristic of the different filter stages can be calculated throughout these components. For this purpose the $\mathbf{A}$-matrices of the filter circuits are used.
After comparing the input signal with the selected minimum and maximum frequencies, the program controls whether the filters are generally useable. On the one hand it is important, that the filter fulfills the required attenuation in the given frequency band and on the other hand it is checked whether the filter matches with the given filter stage. If these two conditions are met, the respective control variable "Count" is set to the value 1 and a specific value for the weight and price for this filter is defined. If the first condition is not fulfilled, the control variable "Count" is set to 0 , the specific cost and the specific weight of the filter are set to the value 100. In the next step, the cost and weight of the filter are sorted by size, starting with the lowest. After that, the five filters with the lowest weight and the five filters with the smallest price will be displayed. Filters with the control variable "Count" $=0$ are not considered.

\subsection{Studied filter circuits}

For the program and the later practical applications, it is necessary to consider different filter circuits. In the simulation program the following passive filter circuits are implemented to the fifth order: LC-, RC-, LRC-, T- and $\pi$-filter. In active filters, operational amplifiers are used. The advantage of using operational amplifiers is the low weight and the low construction volume. The ideal operational amplifier, which is used in these simulations has the following characteristics: The internal impedance of the operational amplifier is infinitely large. The input current $\underline{I}_{\mathrm{e}}$ is zero. The output impedance of the operational amplifier is zero. The voltage gain $A_{\mathrm{u}, \mathrm{d}}$ for differential signals is infinitely large. Therefore the voltage between the input terminals is zero, when the output signal is limited. The common-mode rejection is infinite large.

Previous studies (Rehfeld, 2012) have shown, that an active filter with an inductive coupling and capacitive decoupling has the best filter characteristics. In these previous studies different active filters with operational amplifiers were studied and the the active LC-filter in Fig. 5 was chosen. This filter is used for further considerations. It consists of a transformer, which decouples the interference signal via the inductance $L_{1}$ and transmits it to the inductance $L_{2}$, in the secondary circuit. There, the decoupled signal is inverted by an inverter circuit and is transfered by means of the capacitor $C_{1}$ back to the load circuit.

\subsection{Filter Optimization algorithm}

The optimization of a filter is a complex problem, because filters are described by several parameters, which in addition need to be in a certain solution space. 


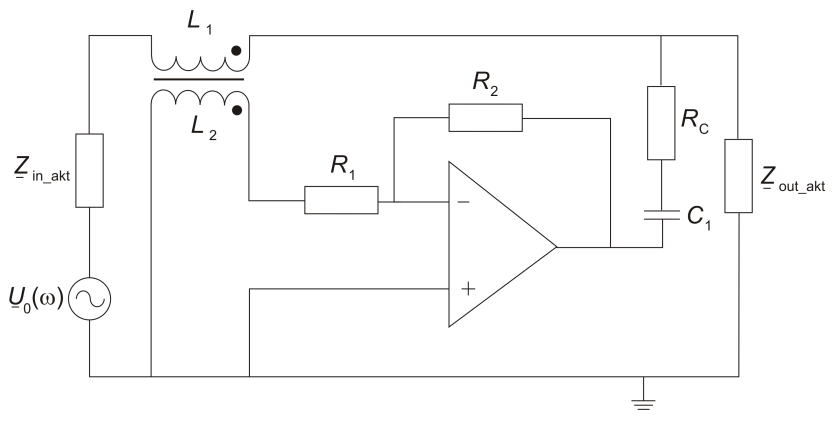

Fig. 5. Active Filter with inductive coupling and capacitance decoupling.

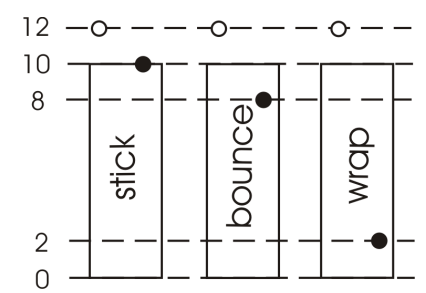

Fig. 6. Restriction condition for the PSO-algorithm (Brodersen, 2007).

\subsubsection{Brute-Force-Method}

The Brute-Force-Method tries each or a majority of all possible solutions meeting the boundary conditions, until a solution is found. The advantage of this method is the determination of an unique solution of the problem. However, the disadvantage is the requirement of high computing time due to an increasing number of variables in the solution space.

\subsubsection{Ant-Colony-Algorithm}

Another way to solve a complex problem is the Ant-ColonyAlgorithm. Here, the natural behavior of ants when foraging is imitated. Each ant has a memory and also the entire ant colony has a collective memory. The ants have several possible routes to reach the feeding place. The result of the algorithm is the optimal way to the food source (Boysen, 2006). This method is used very often for the optimization of business processes. It would also be possible to design filters with this process.

\subsubsection{Particle-Swarm-Optimization}

Another solution method is the Particle-Swarm-Optimization (PSO). This process provided good results in the field of engineering, e.g. in the construction of bandpass filters or the design of gears and can be applied to the current problem. The algorithm will be described more in detail below and is adapted to the problem of filter design.

\section{Mathematical description:}

Basically, the $i$-th particle of a swarm is described by:

- $\boldsymbol{x}_{i}$ : current position of the particle

- $\boldsymbol{v}_{i}$ : current velocity of the particle

- $\boldsymbol{y}_{i}$ : best position of the particle

In addition, a certain fitness function $f\left(\boldsymbol{x}_{i}\right)$ is assumed. From this function we obtain the so called fitness value, that indicates how far the current position is away from the target. From the fitness value the next best position results:

$\boldsymbol{y}_{i}(t+1)=\left\{\begin{array}{cl}\boldsymbol{y}_{i}(t) & \text { if } f\left(\boldsymbol{x}_{i}(t+1)\right)<f\left(\boldsymbol{y}_{i}(t)\right) \\ \boldsymbol{x}_{i}(t+1) & \text { if } f\left(\boldsymbol{x}_{i}(t+1)\right) \geq f\left(\boldsymbol{y}_{i}(t)\right)\end{array}\right.$

The next important parameter of the PSO is the swarm. It consists of $p$ particles. The swarm has a collective memory, which remembers the best position of the particle with the highest fitness value. This value is called the global best position. It is given by:

$$
\begin{gathered}
\boldsymbol{y}_{\mathrm{gb}}(t+1)= \\
= \begin{cases}\boldsymbol{y}_{\mathrm{gb}}(t) & \text { if } f\left(\boldsymbol{y}_{0 \ldots p}(t+1)\right)<f\left(\boldsymbol{y}_{\mathrm{gb}}(t)\right) \\
\left(\boldsymbol{y}_{0 \ldots p}(t+1)\right)_{\max } & \text { if } f\left(\boldsymbol{y}_{0 \ldots p}(t+1)\right) \geq f\left(\boldsymbol{y}_{\mathrm{gb}}(t)\right)\end{cases}
\end{gathered}
$$

From the best position of each particle and the global best position, the new velocity of a particle is results:

$$
\begin{gathered}
\boldsymbol{v}_{i}(t+1)=w \cdot \boldsymbol{v}_{i}(t)+c_{1} r_{1}(t) . \\
{\left[\boldsymbol{y}_{i}(t)-\boldsymbol{x}_{i}(t)\right]+c_{2} r_{2}(t)\left[\boldsymbol{y}_{g b}(t)-\boldsymbol{x}_{i}(t)\right]}
\end{gathered}
$$

With the variables:

$0 \leq c_{1}, c_{2}, w \leq 2$

The two functions $r_{1}(t)$ and $r_{2}(t)$ are random numbers (Brodersen, 2006). After determining the new velocity of each particle, the new current position of the particle results:

$\boldsymbol{x}_{i}(t+1)=\boldsymbol{x}_{i}(t)+\boldsymbol{v}_{i}(t+1) \cdot \Delta t$

This algorithm is repeated until ( $\mathrm{Wu}, 2006)$ :

a. a particle has found the optimal position

b. it is certain, that the optimal position for the solution space does not exist

It is possible that a particle leaves the predetermined solution space. There are three possible restriction conditions for this case. In Fig. 6 the conditions stick, bounce and wrap are 


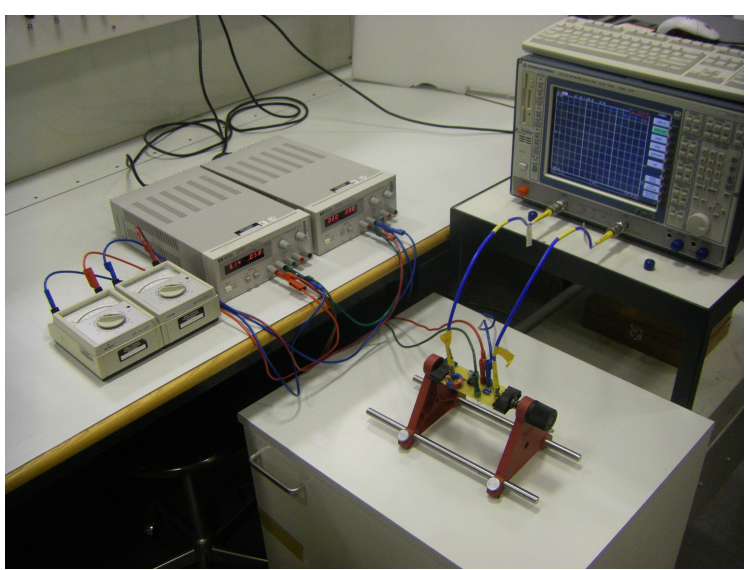

Fig. 7. Measurement setup for measuring the filter response.

shown. For the solution space the numbers from 0 to 10 are set. The white particle leaves the solution space. As a result, the black particle is created in the solution space, according to the following rules.

For the stick specification the particle remains at the maximum value of the solution space. The particle jumps back the amount in the solution space for which he has left it at the bounce condiction. For the wrap specification the particle starts at a lower limit, when leaving the solution space at the upper limit. It is increased by the amount, by which it has left the solution space at the upper limit.

\section{PSO for filter design:}

The PSO algorithm, is now applied to filter design. Since a filter consists of inductors, capacitors and resistors, these components can be regarded as the current position of a particle. Accordingly, the velocity of the particle also results as a vector consisting of a change of the inductance, capacitance and resistance. Thus, the best position of the particle is also a vector which consists of an inductor, a capacitor and a resistor.

$\boldsymbol{x}_{i}=\left(\begin{array}{l}L_{i}(t) \\ C_{i}(t) \\ R_{i}(t)\end{array}\right), \quad \boldsymbol{v}_{i}=\left(\begin{array}{c}\Delta L_{i}(t) \\ \Delta C_{i}(t) \\ \Delta R_{i}(t)\end{array}\right), \quad \boldsymbol{y}_{i}=\left(\begin{array}{l}L_{i}(t) \\ C_{i}(t) \\ R_{i}(t)\end{array}\right)$

After the start of the algorithm for each particle a random position and velocity in the solution space are set. The specific attenuation is determined by means of the $\mathbf{A}$-matrix process described in Sect. 2. With this attenuation the corresponding fitness value of the filter is determined. The fitness value is depending on the attenuation of the filter $D$ in $\mathrm{dB}$. The fitness value is 0 , if the attenuation is smaller than the attenuation set value $-10 \mathrm{~dB}$. From this point the fitness value increases linearly. When the filter reaches the required attenuation $D_{\text {soll }}$, the fitness value will be 1 .

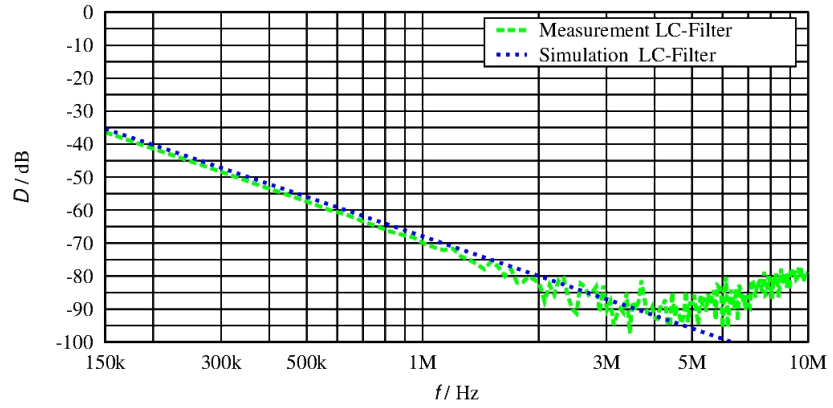

Fig. 8. Comparison of the simulation and measurement results of a passive LC filter.

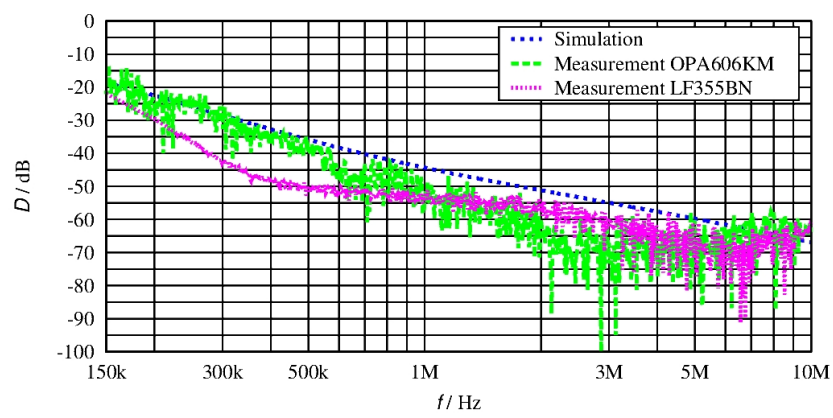

Fig. 9. Comparison of the simulation and measurement results of an active common-mode filter.

Through the fitness value the best position of a particle and the global best position $\boldsymbol{y}_{\mathrm{gb}}$ can be calculated. From these values the new velocity of the particle is determined. This process is repeated until, the optimal solution is found - in this case, the optimal attenuation of the filter.

\section{Validation of the simulation by measurements}

After different filter circuits have been simulated, these should be validated by measurements. For this purpose, different filters have been constructed and measured. The general measurement setup is shown in Fig. 7. The connections of the semi-rigid cables and the filter setup were done using high frequency SMA connectors. The components of the filter circuit were chosen small and with less tolerances so the measurement will be possible in the measured frequency range. The influence of the other cabling could be neglected, because the cabling did not change the measurement results noticeably.

In Fig. 8 the measurement and simulation results of a passive filter are shown. There the attenuation $D$ in $\mathrm{dB}$, is plotted over the frequency $f$ in $\mathrm{Hz}$.

Up to a frequency of $4 \mathrm{MHz}$, the filter responses are almost identical. From an attenuation of $-80 \mathrm{~dB}$ the measurement is not longer accurate, since the signal-to-noise ratio becomes 


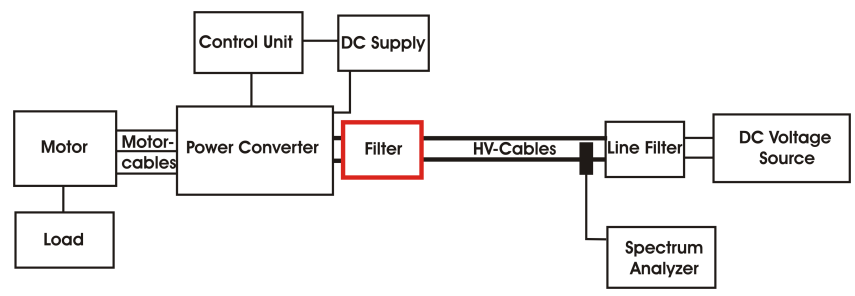

Fig. 10. Measurement setup of the electric powertrain including filter circuit.

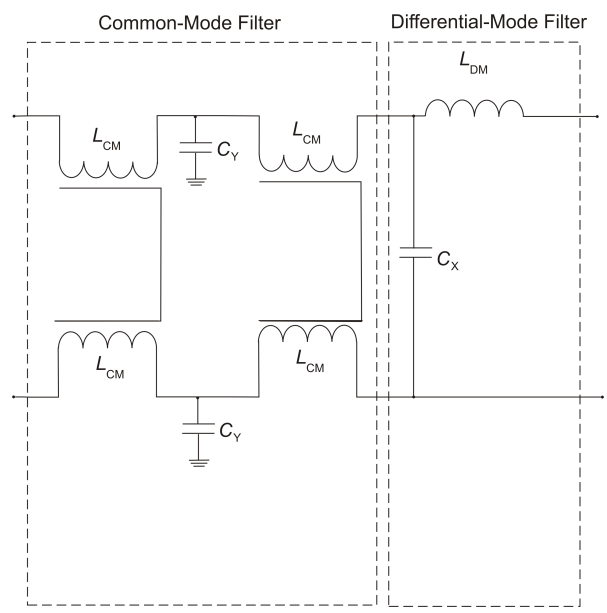

Fig. 11. Used filter circuit at the measurement setup of the electric powertrain.

too small. For this topology, parasitic effects are noticeable once the frequency exceeds about $4 \mathrm{MHz}$. This is explained by the parasitic inductance in series with the capacitor.

Another representative comparison shows the following diagram (Fig.9) of measurement and simulation results of the attenuation of an active common-mode filter. The characteristic of the simulated filter circuit is shown with big dotted blue line. For the measurement setup two different operational amplifiers were used. The reasons for the deviations from the simulations are on the one hand the limited bandwidth of the operational amplifiers and on the other hand the voltage drop between the inverting and noninverting input, which deviates from the simulation.

\section{Practical setup of a filter for the electrical powertrain}

After validating the simulated filter circuits by measurements, the filter is studied in a practical test setup of e.g. an electric powertrain. One possible measurement setup is shown in Fig. 10.

In Fig. 11 the used filter can be seen. The choice of the used components must take the real operating conditions of the filter into account. Magnetic components must be designed to avoid saturation. Moreover, insulation and capacitors must be electrically strong to withstand the occur-

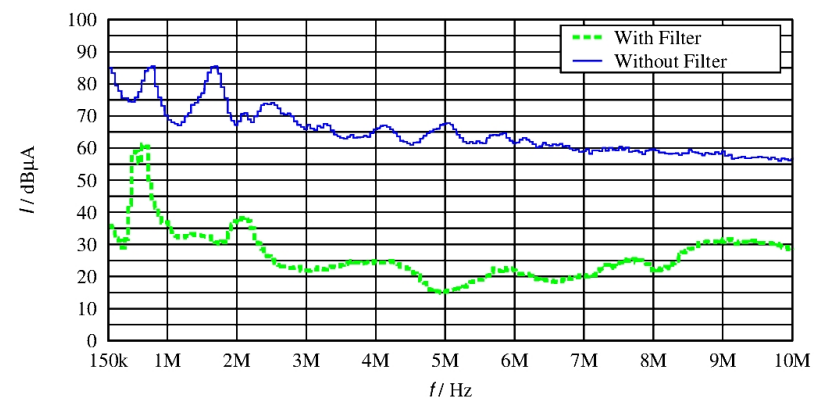

Fig. 12. Comparison of the measurement results with and without a filter.

ing voltages. Please note that this is only one possible filter out of many different usable filter circuits. In this circuit Y-capacitors $C_{\mathrm{Y}}$, common-mode chokes $L_{\mathrm{CM}}$ and a Xcapacitor $C_{\mathrm{X}}$ as well as a differential-mode inductor $L_{\mathrm{DM}}$ are used.

The spectrum is measured with a current measuring clamp connected to a spectrum analyzer. In Fig. 12 a comparison of the interferences on the HV-cables with and without filters is shown.

The filter attenuates the interferences in the entire required frequency range. The lower attenuation at lower frequencies can be increased by using components with lower tolerance.

\section{Conclusions}

In the early development process of EMC filters, simulations are helpful. Due to the large number of possible filter variants, the manual determination is time-consuming. Therefore, a software tool has been developed which determines filters for DC applications quickly, efficiently and optimizes their weight, volume and cost. A further advantage is, that new filter concepts, such as hybrid filter structures can be implemented quickly and compared with conventional concepts. Here, an optimal filter design is determined by using a simulation tool including an effective optimization algorithm. The comparison of simulation and measurement results show good agreement. Investigations with an electrical powertrain setup verified the filter effect.

\section{References}

Boysen, N.: Ameisenalgorithmen, 2006. Universität Hamburg, 2006.

Brodersen, O.: Particle Swarm Optimization in der stochastischen Lagerhaltung, 2006. Gemeinsamer Workshop der Arbeitsgruppen "Entscheidungstheorie und -praxis" und "Fuzzy-Systeme, Neuronale Netze und Künstliche Intelligenz" der Gesellschaft für Operations Research e.V. (GOR) Kaiserslautern, 2006. 
Brodersen, O. and Schumann, M.: Einsatz der Particle Swarm Optimization zur Optimierung universitärer Stundenpläne, 2007, Arbeitsbericht Nr. 05/2007. Universität Göttingen, 2007.

DIN-EN 50178 / VDE 0160: Ausrüstung von Starkstromanlagen mit elektronischen Betriebsmitteln, 1998, DIN Deutsches Institut für Normung e.V. und VDE Verband deutscher Elektrotechniker e.V., 1998

Hagmann, G.: Grundlagen der Elektrotechnik, 2008, 13 Edn., AULA-Verlag, 2008.

Marti, O and Plettl, A.: Physikalische Elektronik und Messtechnik, 2007, Universität Ulm, 2007.
Rehfeld, M.: EMC-Studies for optimal Filter Design in Aircraft and Automotive Applications, 2012, Helmut Schmidt University, University of the Federal Armed Forces Hamburg, 2012.

Schinkel, M.: Entwurf und Simulation aktiver EMV-Filter für dreiphasige drehzahlveränderbare Antriebe, 2009, Technische Universität Berlin, 2009.

Schwab, A. J. and Kürner, W.: Elektromagnetische Verträglichkeit, 2011, 6 Edn., Springer-Verlag, 2011.

Wu, G. and Hansen, V. and Kreysa, E. and Gemünd, H.P.: Optimierung von FSS-Bandpassfiltern mit Hilfe der Schwarmintelligenz (Particle Swarm Optimization), 2006, URSI Adv. Radio Sci., 4, 65-71, 2006,

http://www.adv-radio-sci.net/4/65/2006/. 